https://doi.org/10.15407/ujpe64.6.464

A.A. EREMKO ${ }^{1}$ L. BRIZHIK, ${ }^{1}$ V.M. LOKTEV $^{1,2}$

${ }^{1}$ Bogolyubov Institute for Theoretical Physics, Nat. Acad. of Sci. of Ukraine (14b, Metrolohichna Str., Kyiv 03143, Ukraine)

2 National Technical University of Ukraine "Igor Sikorsky Kyiv Polytechnic Institute"

(37, Peremogy Ave., Kyiv 03056, Ukraine)

\title{
GENERALIZED SPIN-ORBIT INTERACTION AND ITS MANIFESTATION IN TWO-DIMENSIONAL ELECTRON SYSTEMS
}

\begin{abstract}
In frame of Dirac quantum field theory that describes electrons and positrons as elementary excitations of the spinor field, the generalized operator of the spin-orbit interaction is obtained using non-relativistic approximation in the Hamilton operator of the spinor field taking into account the presence of an external potential. This operator is shown to contain a new term in addition to the known ones. By an example of a model potential in the form of a quantum well, it is demonstrated that the Schrödinger equation with the generalized spin-orbit interaction operator describes all spin states obtained directly from the Dirac equation. The dependence of the spin-orbit interaction on the spin states in quasi-two-dimensional systems of electrons localized in a quantum well is analyzed. It is demonstrated that the electric current in the quantum well layer induces the spin polarization of charge carriers near the boundary surfaces of the layer, with the polarization of the charge carriers being opposite at the different surfaces. This phenomenon appears due to the spin-orbit interaction and is known as the spin Hall effect, which was observed experimentally in heterostructures with the corresponding geometry.

Keywords: spin-orbit interaction, Dirac equation, Schrödinger equation, 2D electron gas, quantum well, spin Hall effect.
\end{abstract}

\section{Introduction}

It is generally accepted that both the origin and the magnitude of the spin-orbit interaction (SOI), which occurs in various electron systems, are wellknown and profoundly studied $[1,2]$. In principle, such a viewpoint does not raise objections, and one may agree that SOI is of an exclusively relativistic origin. The concept of SOI was introduced into the Schrödinger equation (SE) on the basis of some empirical (actually, classic) considerations, and it is known as the Thomas correction ${ }^{1}$ [3]. Since the SOI effect is purely relativistic, its exhaustive description

(C) A.A. EREMKO, L. BRIZHIK, V.M. LOKTEV, 2019 can be obtained only in the framework of the Dirac theory. As a rule, the known relativistic corrections to the SE (one of them was called the SOI operator) are obtained by either making a non-relativistic limiting transition in the Dirac equation (DE) or using the method of the approximate Foldy-Wouthuysen transformation of the Dirac Hamiltonian with respect to the small parameter $v / c$, where $v$ is the characteristic velocity of the particle, and $c$ the light speed.

${ }^{1}$ It is worth to recall that this correction was independently and simultaneously proposed by Ya.I. Frenkel [4]. Therefore, it would be proper to call it the Thomas-Frenkel correction.

ISSN 2071-0194. Ukr. J. Phys. 2019. Vol. 64, No. 6 
In spite of that, the authors of works $[5,6]$ made an attempt to obtain the form of the SOI operator on the basis of the direct solution of the DE, in which the external potential was taken into account from the very beginning. Additionally, the attention was paid to the fact that there are several operators these are the so-called spin invariants - that commute with the DE, but not with one another. From whence, it follows that the sought solutions of the DE (even those obtained in the framework of perturbation theory with respect to the same small parameter) can be different. In other words, this means that the DE has a number of solutions, and, hence, there may exist other corrections to the SE. Really, it turned out that the corresponding physical solutions, or the eigenfunctions and eigenvalues, can be determined, which behave differently under different circumstances, i.e. depending on the potential form and shape, the geometry of the system, and so on. However, the eigenvalues and eigenfunctions found in the cited works do not provide an ultimate solution to the problem, because there arises a problem of determining the form of corresponding corrections or the SOI operator in the SE, considering the spin invariants which those solutions correspond to.

This was the aim of this work, in which we relied on the Dirac quantum field theory that describes electrons and positrons as elementary excitations of a spinor field. By applying the non-relativistic approximation to the Hamilton operator of the spinor field with regard for the presence of an external potential, a generalized SOI operator can be found. By an example of a quantum-well (QW) model potential, it is shown that the SE with the generalized SOI operator can describe all spin states obtained directly from the DE itself $[5,6]$. The dependence of SOI on the spin state in quasi-two-dimensional (2D) electron systems localized in the QW plane is analyzed. Recall that the consideration of SOI and the study of its effects in low-dimensional systems (for example, various heterostructures) is a challenging problem of modern theoretical and applied solid-state physics.

\section{Hamiltonian of Particles in External Fields}

In quantum field theory, the Hamiltonian of the Dirac spinor field has the form of the integral $[7,8]$

$\mathrm{H}=\int \mathcal{H} d \mathbf{r}=\int \Psi^{\dagger}(\mathbf{r}, t) \hat{H}_{D} \Psi(\mathbf{r}, t) d \mathbf{r}$, where the integration is carried out over the entire volume, and the four-component function $\Psi(\mathbf{r}, t)=$ $=\left(\psi_{1}: \psi_{2}: \psi_{3}: \psi_{4}\right)^{T}$ (the 4-spinor or bispinor) is the amplitude of the spinor field. The function $\Psi(\mathbf{r}, t)$ satisfes the DE

$i \hbar \frac{\partial \Psi}{\partial t}=\hat{H}_{D} \Psi$

with the Hamiltonian

$\hat{H}_{D}=c\left(\hat{\mathbf{p}}-\frac{e}{c} \mathbf{A}(\mathbf{r})\right) \hat{\boldsymbol{\alpha}}+e \Phi(\mathbf{r}) \hat{I}+m c^{2} \hat{\beta}$.

Here, $\hat{\mathbf{p}}=-i \hbar \boldsymbol{\nabla}$ is the momentum operator for a particle with the mass $m$ and the charge $e ; \hat{\boldsymbol{\alpha}}=\sum_{j} \mathbf{e}_{j} \hat{\alpha}_{j}$ is a vector matrix, whose components $\hat{\alpha}_{j}(j=x, y, z)$ together with the matrix $\hat{\beta}$ and the unit matrix $\hat{I}$ are Hermitian Dirac 4-matrices; and $\mathbf{A}(\mathbf{r})$ and $\Phi(\mathbf{r})$ are the vector and scalar, respectively, potentials of the external electromagnetic field. According to quantum mechanics, the components of the function $\Psi$ are $q$ numbers, i.e. $\psi_{\nu}^{\dagger} \psi_{\mu} \neq \psi_{\mu} \psi_{\nu}^{\dagger}$, where $(\nu, \mu)=1,2,3,4$, and the operator $\hat{H}_{D}$ in Eqs. (1) and (3) is called the Dirac Hamiltonian.

Since an arbitrary bispinor $\Psi(\mathbf{r})$ can be expanded in a complete basis of orthonormal bispinors $\Psi_{\{\nu\}}(\mathbf{r})$,

$\Psi(\mathbf{r})=\sum_{\{\nu\}} \hat{c}_{\{\nu\}} \Psi_{\{\nu\}}(\mathbf{r})$,

then Hamiltonian (1) can easily be written in terms of the creation, $\hat{c}_{\{\nu\}}^{\dagger}$ and annihilation, $\hat{c}_{\{\nu\}}$, operators, which are characterized by a set of quantum numbers $\{\nu\}$. The natural choice for this basis is the eigenbispinors of the equation

$\hat{H}_{D}^{(0)} \Psi^{(0)}(\mathbf{r})=E \Psi^{(0)}(\mathbf{r})$,

where

$\hat{H}_{D}^{(0)}=c \hat{\mathbf{p}} \hat{\boldsymbol{\alpha}}+m c^{2} \hat{\beta}$,

which describe stationary states of free particles in the absence of external fields. In this case, it is convenient to use plane waves

$\Psi(\mathbf{r})=\Psi(\mathbf{k}) \exp (i \mathbf{k r})$

as the eigenfunctions of the operator $\hat{\mathbf{p}}$, which is an integral of motion for problem (4). The components of the wave vector $\mathbf{k}$ determine the eigenvalues of the momentum $\mathbf{p}=\hbar \mathbf{k}$ and belong to the set of quantum numbers $\{\nu\}$. 
If the orthonormalization conditions are imposed, it is easier to work with a discrete spectrum, rather than with a continuous one. With this aim in view, let us confine the space by a cube with the edge length $L$. Then, by imposing the cyclic boundary conditions on the solutions, we arrive at the quasicontinuous spectrum of values for the components of the vector $\mathbf{k}=\sum_{j} k_{j} \mathbf{e}_{j}$ :

$k_{j}=\frac{2 \pi}{L} n_{j}, \quad n_{j}=0, \pm 1, \pm 2, \ldots, \quad j=x, y, z$,

where the numbers $n_{j}$ take all integer values from $-\infty$ to $+\infty$.

Substituting Eq. (5) into Eq. (4), we obtain the matrix equation

$$
\left(\begin{array}{cc}
m c^{2} \hat{I}_{2} & \hbar c \mathbf{k} \hat{\boldsymbol{\sigma}} \\
\hbar c \mathbf{k} \hat{\boldsymbol{\sigma}} & -m c^{2} \hat{I}_{2}
\end{array}\right)\left(\begin{array}{l}
\psi_{u} \\
\psi_{d}
\end{array}\right)=E\left(\begin{array}{l}
\psi_{u} \\
\psi_{d}
\end{array}\right)
$$

where $\hat{\boldsymbol{\sigma}}$ is a vector matrix, whose components are the Pauli matrices, and $\hat{I}_{2}$ is a unit matrix of the second rank. In this case, the eigenbispinor of Eq. (4) looks like $\Psi^{(0)}(\mathbf{k})=\left(\psi_{u}(\mathbf{k}) \psi_{d}(\mathbf{k})\right)^{T}$, where $\psi_{u}=$ $=\left(\psi_{1} \psi_{2}\right)^{T}$ and $\psi_{d}=\left(\psi_{3} \psi_{4}\right)^{T}$ are its upper and lower, respectively, spinors.

It is rather easy to find a solution of Eq. (6), the orthonormalized eigenbispinors of which are given by the expressions $[1,2]$

$$
\begin{aligned}
& \Psi_{e, \sigma}^{(0)}(\mathbf{k})=A_{\mathbf{k}}\left(\begin{array}{c}
\chi_{e, \sigma} \\
\frac{\hbar c \mathbf{k} \cdot \hat{\boldsymbol{\sigma}}}{\varepsilon(\mathbf{k})+m c^{2}} \chi_{e, \sigma}
\end{array}\right), \quad E=\varepsilon(\mathbf{k}), \\
& \Psi_{p, \sigma}^{(0)}(\mathbf{k})=A_{\mathbf{k}}\left(\begin{array}{c}
-\frac{\hbar c \mathbf{k} \cdot \hat{\boldsymbol{\sigma}}}{\varepsilon(\mathbf{k})+m c^{2}} \chi_{p, \sigma} \\
\chi_{p, \sigma}
\end{array}\right), E=-\varepsilon(\mathbf{k}),
\end{aligned}
$$

in which the following notations were introduced:

$A_{\mathbf{k}}=\sqrt{\frac{\varepsilon(\mathbf{k})+m c^{2}}{2 \varepsilon(\mathbf{k})}}, \quad \varepsilon(\mathbf{k})=\sqrt{m^{2} c^{4}+c^{2} \hbar^{2} \cdot \mathbf{k}^{2}}$.

The bispinors $\Psi_{e, \sigma}^{(0)}$ and $\Psi_{p, \sigma}^{(0)}$ in Eq. (7) are the amplitudes of the fields of particles (electrons, $e$ ) and antiparticles (positrons, $p$ ), respectively.

Equation (6) has four eigenvectors, because it is the equation for the eigenvalues of a 4-matrix. Therefore, the number $\sigma$ was introduced in solutions (7). This parameter has two values that are attributed to a pair of orthogonal spinors $\chi_{\nu, \sigma}$ 's: $\chi_{\nu, \sigma}^{\dagger} \chi_{\nu, \sigma^{\prime}}=\delta_{\sigma, \sigma^{\prime}}$. Thus, system (7) determines a complete set of bispinors, which can be used in order to represent an arbitrary bispinor in the form

$\Psi(\mathbf{r})=\frac{1}{L^{3 / 2}} \sum_{\mathbf{k}, \sigma} e^{i \mathbf{k r}}\left(a_{\mathbf{k}, \sigma} \Psi_{e, \sigma}^{(0)}(\mathbf{k})+b_{-\mathbf{k}, \sigma}^{\dagger} \Psi_{p, \sigma}^{(0)}(\mathbf{k})\right)$,

where $a_{\mathbf{k}, \sigma}\left(b_{\mathbf{k}, \sigma}\right)$ and $a_{\mathbf{k}, \sigma}^{\dagger}\left(b_{\mathbf{k}, \sigma}^{\dagger}\right)$ are the operators of the particle (antiparticle) creation and annihilation, respectively. The physical condition of positive definiteness imposed on the eigenvalues of Hamiltonian (1) requires that those operators have to obey the Fermi statistics.

Nevertheless, the definition of bispinors in Eq. (9) is ambiguous, because bispinors (7) satisfy Eq. (6) for arbitrary spinors $\chi_{\nu, \sigma}(\nu=e, p)$. Therefore, the physical meaning of $\sigma$ as a quantum number is absent. Although the values of the quantity $\sigma$ can be defined as \pm 1 (or $\uparrow, \downarrow$ ), their meaning as projections on that or another axis still remains absent, because the directions of those axis are not given, although it is required for the complete determination of the state.

At the same time, the stationary states of the system are known to be characterized by quantum numbers that correspond to a complete set of observed quantities and have definite values. The operators of those quantities, which are called invariants, commute both with the Hamiltonian of the system and with one another. As was said above, for expressions (7), such numbers are the eigenvalues of the operator $\hat{\mathbf{p}}$ or the components of the vector $\mathbf{k}$.

Concerning the spin number, there are several spin invariants in a uniform space [9], which together with the Hamiltonian have a common system of eigenfunctions (7). The substitution of expressions (7) into the equation for the invariant eigenvalues brings about the equations

$\mathbf{u}_{\nu}(\mathbf{k}) \hat{\boldsymbol{\sigma}} \chi_{\nu, \mathbf{k}, \sigma}=\sigma u_{\nu}(\mathbf{k}) \chi_{\nu, \mathbf{k}, \sigma}, u_{\nu}(\mathbf{k})=\left|\mathbf{u}_{\nu}(\mathbf{k})\right|$,

which determine both a pair of orthogonal spinors and a coordinate system, in which they have the simplest form, $\chi_{\uparrow}=\left(\begin{array}{ll}1 & 0\end{array}\right)^{T}$ and $\chi_{\downarrow}=\left(\begin{array}{ll}0 & 1\end{array}\right)^{T}$. The subscripts $\nu$ and $\sigma$ in Eq. (10) take the relevant values: $\nu=e$ or $p$, and $\sigma= \pm 1$. Then the matrices $\mathbf{u}_{\nu}(\mathbf{k}) \hat{\boldsymbol{\sigma}}$ become independent invariants for particles $(\nu=e)$ and an- 
tiparticles $(\nu=p)^{2}$, so that Eq. (10) gives sense to the number $\sigma= \pm 1$ in the operators $a_{\mathbf{k}, \sigma}^{\dagger}$ and $a_{\mathbf{k}, \sigma}$ in Eq. (9) by indicating that their action leads to the creation or annihilation of a particle with a definite spin invariant value at a given $\mathbf{u}_{\nu}(\mathbf{k})$. Every invariant has its own vectors $\mathbf{u}_{\nu}(\mathbf{k})$ with a characteristic dependence on $\mathbf{k}$, the account of which is mandatory. Note that the spin operator itself is not an integral of motion even in a uniform space. Therefore, in the case of states with a given energy, one may talk about the spin only as about the mean value of the corresponding operator.

Since the spin invariants do not commute with one another, only one of them can correspond to the stationary spin state. The presence of several invariants gives rise to an ambiguous choice of the spin state, which is an actual origin of the spinor arbitrariness in Eq. (7).

Important is the fact that the spin state is not defined a priori. In the general case, an arbitrary linear combination of all invariants, whose coefficients are free parameters, can be chosen as such an invariant. The explicit expressions for the vectors $\mathbf{u}_{e}(\mathbf{k})$ and $\mathbf{u}_{p}(\mathbf{k})(\mathrm{k})$ are given in work [10], where it was shown that they really contain free parameters or the components of the vectors $\mathbf{u}_{\boldsymbol{\mu}} \equiv \mathbf{u}_{\boldsymbol{\mu}}(\mathbf{k})$ and $\mathbf{u}_{\mathcal{S}} \equiv \mathbf{u}_{\mathcal{S}}(\mathbf{k})$. The subscripts $\boldsymbol{\mu}$ and $\mathcal{S}$ are associated with the notation for the initial invariants: $\boldsymbol{\mu}$ for the vector of magnetic spin polarization and $\mathcal{S}$ for the vector of spin polarization [9], the linear combination of which $\hat{\mathcal{I}}_{\text {gen }}=\mathbf{r}_{\boldsymbol{\mu}}(\mathbf{k}) \hat{\boldsymbol{\mu}}+\mathbf{r}_{\mathcal{S}}(\mathbf{k}) \hat{\mathcal{S}}$ is an invariant in the general form. In the coordinate space, $\mathbf{k} \rightarrow(1 / \hbar) \hat{\mathbf{p}}$, and the vectors $\mathbf{u}_{\boldsymbol{\mu}}$ and $\mathbf{u}_{\mathcal{S}}$ become operators that commute with the Hamiltonian and with the invariants of the spatial motion. The choice of $\mathbf{u}_{\boldsymbol{\mu}}$ and $\mathbf{u}_{\mathcal{S}}$ that explicitly depend on $\hat{\mathbf{p}}$ corresponds to that or another invariant. For example, if $\mathbf{u}_{\boldsymbol{\mu}}=\hat{\mathbf{p}}$ and $\mathbf{u}_{\mathcal{S}}=0$, we deal with the operator of helicity, whereas if $\mathbf{u}_{\boldsymbol{\mu}}=0$ and $\mathbf{u}_{\mathcal{S}}=\mathbf{e}_{j} \times \hat{\mathbf{p}}$, this is the corresponding component of the vector of electric spin polarization.

\section{Spin-Orbit Interaction}

Expressions (7), in which the spinors are given by Eq. (10) with the vectors $\mathbf{u}_{e}(\mathbf{k})$ and $\mathbf{u}_{p}(\mathbf{k})$, give an explicit form of the bispinors $\Psi_{e, \sigma}^{(0)}(\mathbf{k})$ in expansion

2 In the general case, the vectors $\mathbf{u}_{e}(\mathbf{k})$ and $\mathbf{u}_{p}(\mathbf{k})$ do not coincide.
(9). The substitution of Eq. (9) into Eq. (1) leads to the Hamiltonian of the Dirac spinor field in the representation of free-particle occupation numbers,

$$
\begin{aligned}
& \mathrm{H}=\sum_{\mathbf{k}, \sigma}\left(\varepsilon(\mathbf{k}) a_{\mathbf{k}, \sigma}^{\dagger} a_{\mathbf{k}, \sigma}+\right. \\
& \left.+\frac{1}{L^{3}} \sum_{\mathbf{k}^{\prime}, \sigma^{\prime}} V_{\sigma, \sigma^{\prime}}^{(e-e)}\left(\mathbf{k}, \mathbf{k}^{\prime}\right) a_{\mathbf{k}, \sigma^{\dagger}}^{\dagger} a_{\mathbf{k}^{\prime}, \sigma^{\prime}}\right)+ \\
& +\sum_{\mathbf{k}, \sigma}\left(-\varepsilon(\mathbf{k}) b_{-\mathbf{k}, \sigma} b_{-\mathbf{k}, \sigma}^{\dagger}+\right. \\
& \left.+\frac{1}{L^{3}} \sum_{\mathbf{q}, \sigma^{\prime}} V_{\sigma, \sigma^{\prime}}^{(p-p)}\left(\mathbf{k}, \mathbf{k}^{\prime}\right) b_{-\mathbf{k}, \sigma} b_{-\mathbf{k}^{\prime}, \sigma^{\prime}}^{\dagger}\right)+ \\
& +\frac{1}{L^{3}} \sum_{\mathbf{k}, \mathbf{k}^{\prime}, \sigma, \sigma^{\prime}}\left(V_{\sigma, \sigma^{\prime}}^{(e-p)}\left(\mathbf{k}, \mathbf{k}^{\prime}\right) a_{\mathbf{k}, \sigma}^{\dagger} b_{-\mathbf{k}^{\prime}, \sigma^{\prime}}^{\dagger}+\right. \\
& \left.+V_{\sigma, \sigma^{\prime}}^{(p-e)}\left(\mathbf{k}, \mathbf{k}^{\prime}\right) b_{-\mathbf{k}, \sigma} a_{\mathbf{k}^{\prime}, \sigma^{\prime}}\right)
\end{aligned}
$$

where

$$
\begin{aligned}
& V_{\sigma, \sigma^{\prime}}^{\left(\nu-\nu^{\prime}\right)}\left(\mathbf{k}, \mathbf{k}^{\prime}\right)=\left(\Psi_{\nu, \sigma}^{(0)}(\mathbf{k})\right)^{\dagger} \times \\
& \times\left(V\left(\mathbf{k}-\mathbf{k}^{\prime}\right) \hat{I}-\frac{e}{c} \mathbf{A}\left(\mathbf{k}-\mathbf{k}^{\prime}\right) \hat{\boldsymbol{\alpha}}\right) \Psi_{\nu^{\prime}, \sigma^{\prime}}^{(0)}\left(\mathbf{k}^{\prime}\right)
\end{aligned}
$$

are the particle-particle $\left(\nu=\nu^{\prime}=e\right)$, antiparticleantiparticle $\left(\nu=\nu^{\prime}=p\right)$, and particle-antiparticle $\left(\nu \neq \nu^{\prime}\right)$ scattering matrices, which contain the Fourier images of the scalar, $V(\mathbf{r})$, and vector, $\mathbf{A}(\mathbf{r})$, potentials, i.e. $V(\mathbf{q})$ and $\mathbf{A}(\mathbf{q})$, respectively. Then, operator (11) becomes the sum $\mathrm{H}=\mathrm{H}_{e}+\mathrm{H}_{p}+\mathrm{V}_{e-p}$ of three terms: the particle, $\mathrm{H}_{e}$, and antiparticle, $\mathrm{H}_{p}$, Hamiltonians, and the operator $\mathrm{V}_{e-p}$ of direct particle-antiparticle transformation. If the product of the creation and annihilation operators is in the normal form, Hamiltonian (11) becomes positively determined, except for an infinite additive constant, which is the energy of the state without any particles ("vacuum"). This is the energy, from which the energies of all elementary excitations are reckoned from.

The state of the system with the given number of elementary excitations is described by the ket vector $|\psi\rangle$ that satisfies the $\mathrm{SE}$

$i \hbar \frac{\partial}{\partial t}|\psi(t)\rangle=\mathrm{H}|\psi(t)\rangle$.

Here, in accordance with the considered problem and the conservation laws, the state $|\psi\rangle$ is generated by 
the products of the required numbers of the particle and antiparticle creation operators that act on the vacuum state $|0\rangle$. In a uniform isotropic space, Hamiltonian (11) has a diagonal form and is the sum of the Hamiltonians of free particles and antiparticles. In addition, it also separates the Hamiltonians for particles with opposite spins. As one can see, in the presence of external fields, (i) the independence of the free particle and antiparticle states disappears, and (ii) the elements of the scattering matrix (12) are not only generated by the scalar and vector potentials, but also depend on the form of spinors $\chi_{\nu, \mathbf{k}, \sigma}$ in amplitudes (7). The explicit dependence of spinors (10) on the wave vector points directly at the interrelation between the spin and spatial levels of the particle degrees of freedom. For the description of this interrelation, the SOI concept was introduced. We should emphasize that, hence, SOI is nothing else, but a direct result of the presence of that or another external potential violating the uniform rectilinear motion of particles.

Below, we confine the consideration to the case where the magnetic field is absent, by putting $\mathbf{A}(\mathbf{r})=$ 0 in Eq. (12). As was shown in work [10], in the case of non-relativistic potentials, where the inequality $|V(\mathbf{r})| / m c^{2} \ll 1$ is obeyed, the particle and antiparticle states can be approximately separated with a given accuracy making use of the canonical Schrieffer-Wolf transformation method. In the majority of physical problems, the kinetic energy is also a non-relativistic quantity, so that another inequality, $\hbar k / m c \ll 1$, is obeyed, which allows energies (8) and bispinor convolutions in Hamiltonian (11) to be expanded in series in this small parameter. The approximate renormalization with respect to those both parameters makes it possible to change to the non-relativistic approximation in Hamiltonian (11) and represent it as the sum $\mathrm{H}=\tilde{\mathrm{H}}_{e}+\tilde{\mathrm{H}}_{p}$, where the components $\tilde{\mathrm{H}}_{\nu=e, p}$ describe already independent (quasi)particles and (quasi)antiparticles, which include a small (within the second order of magnitude) "admixture" of the initial states of both particles and antiparticles.

Below, only the electron Hamiltonian is considered, so that the subscript " $e$ " is omitted. The analysis of the antiparticle case is almost identical to the presented one. In particular, in work [10], both parameters were assumed to be of the same order of magnitude, and a non-relativistic approximation for Hamil- tonian (11) was obtained within the second-order corrections. Thus, it was shown that the non-relativistic Hamiltonian $\tilde{\mathrm{H}}_{e}$ for electrons in an external scalar potential looks like

$$
\begin{aligned}
& \tilde{\mathrm{H}}_{e} \equiv \mathrm{H} \simeq \sum_{\mathbf{k}, \sigma}\left(\frac{\hbar^{2} \mathbf{k}^{2}}{2 m}\left(1-\lambda_{\mathrm{SO}} k^{2}\right) a_{\mathbf{k}, \sigma}^{\dagger} a_{\mathbf{k}, \sigma}+\right. \\
& \left.+\frac{1}{L^{3}} \sum_{\mathbf{k}^{\prime}, \sigma^{\prime}} V_{\sigma, \sigma^{\prime}}\left(\mathbf{k}, \mathbf{k}^{\prime}\right) a_{\mathbf{k}, \sigma^{\dagger}}^{\dagger} a_{\mathbf{k}^{\prime}, \sigma^{\prime}}\right),
\end{aligned}
$$

where

$V_{\sigma, \sigma^{\prime}}\left(\mathbf{k}, \mathbf{k}^{\prime}\right)=V\left(\mathbf{k}-\mathbf{k}^{\prime}\right) \chi_{\sigma}^{\dagger} \times$

$\times\left(1-\frac{\lambda_{\mathrm{SO}}}{2}\left(\mathbf{k}-\mathbf{k}^{\prime}\right)^{2}+i \lambda_{\mathrm{SO}} \boldsymbol{\Lambda}\left(\mathbf{k}, \mathbf{k}^{\prime}\right) \hat{\boldsymbol{\sigma}}\right) \chi_{\sigma^{\prime}}$

and the parameter

$\lambda_{\mathrm{SO}}=\frac{\hbar^{2}}{4 m^{2} c^{2}}$

characterizes the order of relativistic corrections and determines the SOI magnitude.

Hamiltonian (13) includes all relativistic corrections of the second order to both the kinetic and potential energies. The second term in the renormalized scattering potential (14) is known as the Darwin correction, and the matrix $\boldsymbol{\Lambda}\left(\mathbf{k}, \mathbf{k}^{\prime}\right) \hat{\boldsymbol{\sigma}}$ is a relativistic correction that is called the SOI operator. In the SOI operator, the vector $\boldsymbol{\Lambda}\left(\mathbf{k}, \mathbf{k}^{\prime}\right)$ is described by the expression

$\boldsymbol{\Lambda}\left(\mathbf{k}, \mathbf{k}^{\prime}\right)=\mathbf{k} \times \mathbf{k}^{\prime}+\boldsymbol{\Lambda}_{\mathrm{BEL}}\left(\mathbf{k}, \mathbf{k}^{\prime}\right)$.

Here, the term $\mathbf{k} \times \mathbf{k}^{\prime}$ corresponds to the ThomasFrenkel correction (see, e.g., works $[1,2,8]$ ). The other term, $\boldsymbol{\Lambda}_{\mathrm{BEL}}\left(\mathbf{k}, \mathbf{k}^{\prime}\right)$, is given by the expression

$\boldsymbol{\Lambda}_{\mathrm{BEL}}\left(\mathbf{k}, \mathbf{k}^{\prime}\right)=\boldsymbol{\Lambda}(\mathbf{k})-\boldsymbol{\Lambda}\left(\mathbf{k}^{\prime}\right)$,

where the vector

$\boldsymbol{\Lambda}(\mathbf{k})=\mathbf{e} \times \mathbf{u}^{(2)}(\mathbf{k})+\frac{\mathbf{e} \cdot\left[\mathbf{e}_{z} \times \mathbf{u}^{(2)}(\mathbf{k})\right]}{1+\mathbf{e e}_{z}} \mathbf{e}$

was also obtained in work [10] in the case where $\mathbf{u}_{e}(\mathbf{k}) \equiv \mathbf{u}(\mathbf{k})$ has the form

$\mathbf{u}(\mathbf{k}) \simeq \mathbf{u}^{(0)}+2 \lambda_{\mathrm{SO}} \mathbf{u}^{(2)}(\mathbf{k})+\ldots$.

In this expansion, $\mathbf{u}^{(0)}$ and $\mathbf{u}^{(2)}$ denote the terms of the zeroth and second orders, respectively:

$\mathbf{u}^{(0)}=\mathbf{u}_{\boldsymbol{\mu}}+\mathbf{u}_{\mathcal{S}}$

ISSN 2071-0194. Ukr. J. Phys. 2019. Vol. 64, No. 6 
$\mathbf{u}^{(2)}(\mathbf{k})=\left(\mathbf{u}_{\mathcal{S}} \cdot \mathbf{k}\right) \mathbf{k}-\left[\mathbf{u}_{\boldsymbol{\mu}} \times \mathbf{k}\right] \times \mathbf{k}$.

Making allowance for them, the solution of Eq. (10) can be written as follows:

$\chi_{\mathbf{k}, \sigma} \simeq \chi_{\sigma}+\lambda_{\mathrm{SO}} \chi_{\mathbf{k}, \sigma}^{(2)}=\left(1-i \lambda_{\mathrm{SO}} \boldsymbol{\Lambda}(\mathbf{k}) \hat{\boldsymbol{\sigma}}\right) \chi_{\sigma}$,

where $\boldsymbol{\Lambda}(\mathbf{k})$ is given by expression (18), and $\chi_{\sigma}$ satisfies the spinor equation

$\mathbf{u}^{(0)} \hat{\boldsymbol{\sigma}} \chi_{\sigma}=\sigma u^{(0)} \chi_{\sigma}, \quad \sigma= \pm 1$.

The guiding cosines $\gamma_{j}$ of the vector $\mathbf{u}^{(0)}$ with respect to the axes of the selected coordinate frame $\left(\sum_{j} \gamma_{j}^{2}=\right.$ 1) are spin variables. For example, it is easy to see that the spinors

$\chi_{\sigma} \equiv \chi_{\sigma}(\theta, \phi)=e^{i \sigma \phi / 2}\left(\begin{array}{c}\sigma \sqrt{\frac{1+\sigma \gamma_{z}}{2}} e^{-i \phi / 2} \\ \sqrt{\frac{1-\sigma \gamma_{z}}{2}} e^{i \phi / 2}\end{array}\right)$,

where

$\tan \phi=\frac{\gamma_{y}}{\gamma_{x}}, \quad \gamma_{z}=\cos \theta$

are the solutions of Eq. (20) in the laboratory coordinate frame $(j=x, y, z)$. In this case, the unit vector $\mathbf{e}=\mathbf{u}^{(0)} / u^{(0)}$ in expression (18) can be written in the parametric form (in terms of spin variables, which are the arguments of spinors (21)) as follows:

$\mathbf{e}\left(\mathbf{k}_{\perp}\right)=\sin \theta \cos \phi \mathbf{e}_{x}+\sin \theta \sin \phi \mathbf{e}_{y}+\cos \theta \mathbf{e}_{z}$.

In view of expression (18), the vector $\boldsymbol{\Lambda}\left(\mathbf{k}, \mathbf{k}^{\prime}\right)$, which characterizes SOI in the non-relativistic Hamiltonian, can be written in the form

$\boldsymbol{\Lambda}\left(\mathbf{k}, \mathbf{k}^{\prime}\right)=\mathbf{k} \times \mathbf{k}^{\prime}+\mathbf{e} \times \boldsymbol{\lambda}\left(\mathbf{k}, \mathbf{k}^{\prime}\right)+\frac{\mathbf{e} \cdot\left[\mathbf{e}_{z} \times \boldsymbol{\lambda}\left(\mathbf{k}, \mathbf{k}^{\prime}\right)\right]}{1+\mathbf{e e}_{z}} \mathbf{e}$

where $\boldsymbol{\lambda}\left(\mathbf{k}, \mathbf{k}^{\prime}\right)=\mathbf{u}^{(2)}(\mathbf{k})-\mathbf{u}^{(2)}\left(\mathbf{k}^{\prime}\right)$, and the vector $\mathbf{u}^{(2)}(\mathbf{k})$ is defined in Eq. (19).

Note that the operators $a_{\mathbf{k}, \sigma}^{\dagger}$ and $a_{\mathbf{k}^{\prime}, \sigma}$ in Eq. (13) are related to the spinors $\chi_{\sigma}$, which satisfy equality (20), and describe the creation and annihilation, respectively, of electrons with the spin polarization determined by the vector $\mathbf{u}^{(0)}$ [see Eq. (19)]. As a result, the vector e that enters expression (23) inserts not only the dependence on spatial variables into the SOI operator, but also the explicit dependence on the spin degrees of freedom. In the external potential, the stationary electron states will be realized only provided a known spin invariant. In the non-relativistic approximation, this is the matrix $\mathbf{u}^{(0)} \hat{\boldsymbol{\sigma}}$. According to the symmetry of the given potential, this spin invariant is related to the vectors $\mathbf{u}_{\boldsymbol{\mu}}$ and $\mathbf{u}_{\mathcal{S}}$ with a dependence on $\mathbf{k}$ (in the coordinate representation, on the momentum), which is to be specified.

\section{Diagonalization of the Hamiltonian with regard for the Spin-Orbit Interaction}

It is well known that the relativistic effects in the problems of non-relativistic physics can be taken into account by adding only the relativistic SOI operator, which describes spin-dependent phenomena, to the standard Schrödinger Hamiltonian. Bearing all that in mind and taking Eqs. (14) and (23) into account, let us write down Hamiltonian (13) in the form

$$
\begin{aligned}
& \mathrm{H}=\sum_{\mathbf{k}, \sigma}\left(\frac{\hbar^{2} \mathbf{k}^{2}}{2 m} a_{\mathbf{k}, \sigma}^{\dagger} a_{\mathbf{k}, \sigma}+\right. \\
& +\frac{1}{L^{3}} \sum_{\mathbf{k}^{\prime}} V\left(\mathbf{k}-\mathbf{k}^{\prime}\right) a_{\mathbf{k}, \sigma}^{\dagger} a_{\mathbf{k}^{\prime}, \sigma}+ \\
& +i \frac{1}{L^{3}} \lambda_{\mathrm{SO}} \sum_{\mathbf{k}^{\prime}, \sigma^{\prime}} V\left(\mathbf{k}-\mathbf{k}^{\prime}\right) \times \\
& \left.\times \chi_{\sigma}^{\dagger} \boldsymbol{\Lambda}\left(\mathbf{k}, \mathbf{k}^{\prime}\right) \hat{\boldsymbol{\sigma}} \chi_{\sigma^{\prime}} a_{\mathbf{k}, \sigma}^{\dagger} a_{\mathbf{k}^{\prime}, \sigma^{\prime}}\right) .
\end{aligned}
$$

As concerning the eigenstates $|\psi(t)\rangle=$ $=\exp (-i \mathcal{E} t / \hbar)|\psi\rangle$ of electrons in a given potential, the solution procedure of the $\mathrm{SE} \mathrm{H}|\psi\rangle=\mathcal{E}|\psi\rangle$ is reduced to the diagonalization of the Hamiltonian with the help of a unitary transformation

$a_{\mathbf{k}, \sigma}=\sum_{\{n\}} \psi_{\{n\}, \sigma}(\mathbf{k}) a_{\{n\}, \sigma}$,

whose coefficients $\psi_{\{n\}, \sigma}(\mathbf{k})$ are determined by an equation for eigenvalues and satisfy the orthonormalization condition

$\sum_{\mathbf{k}} \psi_{\{n\}, \sigma}^{*}(\mathbf{k}) \psi_{\left\{n^{\prime}\right\}, \sigma}(\mathbf{k})=\delta_{\{n\},\left\{n^{\prime}\right\}}$.

These coefficients are given by a set $\{n\}$ of all quantum numbers that determine the energy $E_{\{n\}, \sigma}$, i.e. 
by the equation

$$
\begin{aligned}
& \frac{\hbar^{2} \mathbf{k}^{2}}{2 m} \psi_{\sigma}(\mathbf{k})+\frac{1}{L^{3}} \sum_{\mathbf{k}^{\prime}} V\left(\mathbf{k}-\mathbf{k}^{\prime}\right) \psi_{\sigma}\left(\mathbf{k}^{\prime}\right)+ \\
& +i \lambda_{\mathrm{SO}} \frac{1}{L^{3}} \sum_{\mathbf{k}^{\prime}, \sigma^{\prime}} V\left(\mathbf{k}-\mathbf{k}^{\prime}\right) \chi_{\sigma}^{\dagger} \boldsymbol{\Lambda}\left(\mathbf{k}, \mathbf{k}^{\prime}\right) \times \\
& \times \hat{\boldsymbol{\sigma}} \chi_{\sigma^{\prime}} \psi_{\sigma^{\prime}}\left(\mathbf{k}^{\prime}\right)=\mathcal{E} \psi_{\sigma}(\mathbf{k}) .
\end{aligned}
$$

While solving Eq. (26), it is convenient to change to the coordinate representation,

$\psi_{\sigma}(\mathbf{r})=\frac{1}{L^{3 / 2}} \sum_{\mathbf{k}} e^{i \mathbf{k r}} \psi_{\sigma}(\mathbf{k})$.

Then, the substitution $\mathbf{k} \rightarrow \mathbf{k}^{\prime}+\mathbf{q}$ has to be made in the terms that contain $V\left(\mathbf{k}-\mathbf{k}^{\prime}\right)$ and the double sum over $\mathbf{k}$ and $\mathbf{k}^{\prime}$. As a result, we obtain the equality [see Eq. (23)]

$$
\begin{aligned}
& \boldsymbol{\lambda}\left(\mathbf{k}, \mathbf{k}^{\prime}\right) \equiv \boldsymbol{\lambda}\left(\mathbf{q}, \mathbf{k}^{\prime}\right)=\left(\mathbf{u}_{\mathcal{S}} \cdot \mathbf{q}\right) \mathbf{k}^{\prime}+ \\
& +\left(\mathbf{u}_{\mathcal{S}} \cdot \mathbf{k}^{\prime}\right) \mathbf{q}\left[\mathbf{u}_{\boldsymbol{\mu}} \times \mathbf{q}\right] \times \\
& \times \mathbf{k}^{\prime}-\left[\mathbf{u}_{\boldsymbol{\mu}} \times \mathbf{k}^{\prime}\right] \times \mathbf{q}+\mathbf{u}^{(2)}(\mathbf{q}),
\end{aligned}
$$

where $\mathbf{u}^{(2)}(\mathbf{q})$ is defined in Eq. (19). Finally, Eq. (26) rewritten in the coordinate representation is transformed into a system of SEs for the spin states,

$$
\begin{aligned}
& \left(\frac{\hat{\mathbf{p}}^{2}}{2 m}+V(\mathbf{r})\right) \psi_{\sigma}(\mathbf{r})+i \lambda_{\mathrm{SO}} \times \\
& \times \sum_{\sigma^{\prime}} \chi_{\sigma}^{\dagger} \boldsymbol{\Lambda}(\hat{\mathbf{p}}, \nabla V(\mathbf{r})) \hat{\boldsymbol{\sigma}} \chi_{\sigma^{\prime}} \psi_{\sigma^{\prime}}(\mathbf{r})=\mathcal{E} \psi_{\sigma}(\mathbf{r}),
\end{aligned}
$$

where

$$
\begin{aligned}
& \boldsymbol{\Lambda}(\hat{\mathbf{p}}, \nabla V(\mathbf{r}))=-\frac{i}{\hbar} \nabla V(\mathbf{r}) \times \hat{\mathbf{p}}+\mathbf{e} \times \\
& \times \boldsymbol{\lambda}(\hat{\mathbf{p}}, \nabla V(\mathbf{r}))-\frac{[\mathbf{e} \times \boldsymbol{\lambda}(\hat{\mathbf{p}}, \nabla V(\mathbf{r}))] \mathbf{e}_{z}}{1+\mathbf{e e}_{z}} \mathbf{e} .
\end{aligned}
$$

In the last expression, the following notations were introduced:

$$
\begin{aligned}
& \boldsymbol{\lambda}(\hat{\mathbf{p}}, \nabla V(\mathbf{r}))=-\frac{i}{\hbar} \hat{\boldsymbol{\lambda}}-\boldsymbol{\lambda}(\boldsymbol{\nabla} V(\mathbf{r})), \\
& \hat{\boldsymbol{\lambda}}=\left(\mathbf{u}_{\mathcal{S}} \cdot \nabla V(\mathbf{r})\right) \hat{\mathbf{p}}+\nabla V(\mathbf{r})\left(\mathbf{u}_{\mathcal{S}} \cdot \hat{\mathbf{p}}\right)+ \\
& +\left[\nabla V(\mathbf{r}) \times \mathbf{u}_{\boldsymbol{\mu}}\right] \times \hat{\mathbf{p}}+\nabla V(\mathbf{r}) \times\left[\mathbf{u}_{\boldsymbol{\mu}} \times \hat{\mathbf{p}}\right] \\
& \boldsymbol{\lambda}(\boldsymbol{\nabla} V(\mathbf{r}))=\nabla\left(\mathbf{u}_{\mathcal{S}} \cdot \nabla V(\mathbf{r})\right)+ \\
& +\nabla \times\left[\mathbf{u}_{\boldsymbol{\mu}} \times \nabla V(\mathbf{r})\right] .
\end{aligned}
$$

Transformation (25) with the coefficients determined by Eq. (28) brings Hamiltonian (24) to the diagonal form with respect to the spatial degrees of freedom, and the diagonalization with respect to the spin number means the determination of spinors $\chi_{\sigma}(\theta, \phi)$ satisfying the condition $\chi_{\sigma}^{\dagger} \boldsymbol{\Lambda}(\hat{\mathbf{p}}) \hat{\boldsymbol{\sigma}} \chi_{\sigma^{\prime}} \sim \delta_{\sigma, \sigma^{\prime}}$. This relationship holds true, if the spinors $\chi_{\sigma}$, which are eigenfunctions for the matrix e $\hat{\boldsymbol{\sigma}}$ according to definition (20), are also eigenfunctions for the matrix $\boldsymbol{\Lambda}(\hat{\mathbf{p}}) \hat{\boldsymbol{\sigma}}$. This is possible, if these mutually independent matrices commute, namely, if

$[\mathbf{e} \hat{\boldsymbol{\sigma}}, \boldsymbol{\Lambda}(\hat{\mathbf{p}}) \hat{\boldsymbol{\sigma}}]=\mathbf{e} \boldsymbol{\Lambda}(\hat{\mathbf{p}})-\boldsymbol{\Lambda}(\hat{\mathbf{p}}) \mathbf{e}+$

$+i(\mathbf{e} \times \boldsymbol{\Lambda}(\hat{\mathbf{p}})-\boldsymbol{\Lambda}(\hat{\mathbf{p}}) \times \mathbf{e}) \hat{\boldsymbol{\sigma}}=0$.

Equality (31) evidently demands that the vectors e and $\boldsymbol{\Lambda}(\hat{\mathbf{p}})$ should commute with each other. According to Eq. (30), the vector $\boldsymbol{\lambda}(\hat{\mathbf{p}})$ has the term $\boldsymbol{\lambda}(\boldsymbol{\nabla} V(\mathbf{r}))$, which depends on coordinates. Hence, the same term also enters the SOI vector (29). Therefore, the commutation condition for the vector operator $\boldsymbol{\Lambda}(\hat{\mathbf{p}})$ and the unit vector e (generally speaking, this vector in the coordinate representation is also an operator that contains $\hat{\mathbf{p}}$ ) is obeyed provided that $\boldsymbol{\lambda}(\hat{\mathbf{p}})$ does not include a vector that would depend on the spatial coordinates. This requirement brings us to the condition

$$
\begin{aligned}
& \lambda(\boldsymbol{\nabla} V(\mathbf{r}))=\nabla\left(\mathbf{u}_{\mathcal{S}} \cdot \nabla V(\mathbf{r})\right)+ \\
& +\nabla \times\left[\mathbf{u}_{\boldsymbol{\mu}} \times \nabla V(\mathbf{r})\right]=0
\end{aligned}
$$

This equality imposes restrictions on the vectors $\mathbf{u}_{\boldsymbol{\mu}}$ and $\mathbf{u}_{\mathcal{S}}$, and it can be satisfied, only if

$\mathbf{r}_{\mathcal{S}} \cdot \nabla V(\mathbf{r})=0, \quad \mathbf{r}_{\boldsymbol{\mu}} \times \nabla V(\mathbf{r})=0$,

By essence, these are equations for the vectors $\mathbf{r}_{\boldsymbol{\mu}}$ and $\mathbf{r}_{\mathcal{S}}$, and their solutions depend on the specific field symmetry, which reveals itself in the field gradient $\nabla V(\mathbf{r})$.

Since the gradient vector is directed along the normal to the equipotential surface at any of its points, the equality $\nabla V(\mathbf{r})=|\boldsymbol{\nabla} V(\mathbf{r})| \mathbf{n}$ holds true, where $\mathbf{n}$ is a unit normal vector. Then, at every point $M$ in the space, the orthogonal basis $\left\{\mathbf{e}_{1}(M), \mathbf{e}_{2}(M), \mathbf{n}(M)\right\}$, where $\mathbf{e}_{1}(M)$ and $\mathbf{e}_{2}(M)$ are two orthogonal unit vectors that lie in a plane tangent to the equipotential surface at the point $M$ [all three vectors are coupled by the relationship $\mathbf{e}_{1}(M) \times \mathbf{e}_{2}(M)=\mathbf{n}(M)$ ], can

ISSN 2071-0194. Ukr. J. Phys. 2019. Vol. 64, No. 6 
be used. Accordingly, an arbitrary vector a can be expanded in this basis, $\mathbf{a}=\alpha_{1} \mathbf{e}_{1}(M)+\alpha_{2} \mathbf{e}_{2}(M)+$ $+\alpha_{3} \mathbf{n}(M)$, which is nothing else but an expression for this vector in the curvilinear coordinate frame that is related to the potential $V(\mathbf{r})$. According to condition (32), we obtain

$\mathbf{u}_{\mathcal{S}}=\alpha_{1} \mathbf{e}_{1}(M)+\alpha_{2} \mathbf{e}_{2}(M), \quad \mathbf{u}_{\boldsymbol{\mu}}=\alpha_{3} \mathbf{n}(M)$

in this coordinate frame, so that

$\mathbf{e}=\mathbf{u}_{\boldsymbol{\mu}}+\mathbf{u}_{\mathcal{S}}=\alpha_{1} \mathbf{e}_{1}(M)+\alpha_{2} \mathbf{e}_{2}(M)+\alpha_{3} \mathbf{n}(M)$,

where $\alpha_{1}^{2}+\alpha_{2}^{2}+\alpha_{3}^{2}=1$.

Because of the imposed condition, expression (29) contains the equality $\boldsymbol{\lambda}(\hat{\mathbf{p}}, \boldsymbol{\nabla} V)=-(i / \hbar) \hat{\boldsymbol{\lambda}}$, where, according to Eq. (30),

$$
\begin{aligned}
& \hat{\boldsymbol{\lambda}}=\nabla V\left(\mathbf{u}_{\mathcal{S}} \cdot \hat{\mathbf{p}}\right)+\nabla V \times\left[\mathbf{u}_{\boldsymbol{\mu}} \times \hat{\mathbf{p}}\right]= \\
& =|\boldsymbol{\nabla} V(\mathbf{r})|\left\{\mathbf{n}(M)\left(\mathbf{u}_{\mathcal{S}} \cdot \hat{\mathbf{p}}\right)+\right. \\
& \left.+\mathbf{u}_{\boldsymbol{\mu}}(\mathbf{n}(M) \cdot \hat{\mathbf{p}})-\hat{\mathbf{p}}\left(\mathbf{n}(M) \cdot \mathbf{u}_{\boldsymbol{\mu}}\right)\right\} .
\end{aligned}
$$

Now, with the help of definition (33) and the rules of vector computation, we obtain

$\hat{\boldsymbol{\lambda}}=|\nabla V(\mathbf{r})| \mathbf{e} \times(\mathbf{n}(M) \times \hat{\mathbf{p}})=\mathbf{e} \times(\boldsymbol{\nabla} V(\mathbf{r}) \times \hat{\mathbf{p}})$.

In view of this formula and the equality $\mathbf{e}^{2}=1$, vector (29), which characterizes SOI in Eqs. (28), reads

$\boldsymbol{\Lambda}(\hat{\mathbf{p}}, \nabla V)=-\frac{i}{\hbar} \frac{\left(\mathbf{e}+\mathbf{e}_{z}\right) \cdot \nabla V(\mathbf{r}) \times \hat{\mathbf{p}}}{1+\mathbf{e e}_{z}} \mathbf{e}$.

Hence, condition (31) is obeyed automatically for the vectors $\mathbf{u}_{\boldsymbol{\mu}}$ and $\mathbf{u}_{\mathcal{S}}$ that satisfy relations (32), and the matrix element $\chi_{\sigma}^{\dagger} \boldsymbol{\Lambda}(\hat{\mathbf{p}}) \hat{\boldsymbol{\sigma}} \chi_{\sigma^{\prime}} \sim \delta_{\sigma, \sigma^{\prime}}$. A direct consequence of all that is the separation of SE (28) into equations that are independent for every spin state:

$\left(\frac{\hat{\mathbf{p}}^{2}}{2 m}+V(\mathbf{r})+\sigma \frac{\lambda_{\mathrm{SO}}}{\hbar} \frac{\left(\mathbf{e}+\mathbf{e}_{z}\right) \cdot \nabla V(\mathbf{r}) \times \hat{\mathbf{p}}}{1+\mathbf{e e}_{z}}\right) \times$

$\times \psi_{\sigma}(\mathbf{r})=\mathcal{E} \psi_{\sigma}(\mathbf{r})$.

Their solutions $\psi_{\{n\}, \sigma}(\mathbf{r})$ together with the corresponding eigenvalues $E_{\{n\}, \sigma}$ determine the constants in transformation (25),

$\psi_{\{n\}, \sigma}(\mathbf{k})=\frac{1}{L^{3 / 2}} \int e^{-i \mathbf{k r}} \psi_{\{n\}, \sigma}(\mathbf{r}) d \mathbf{r}$.

ISSN 2071-0194. Ukr. J. Phys. 2019. Vol. 64, No. 6
At the same time, Hamiltonian (24) becomes completely diagonalized,

$\mathrm{H}=\sum_{\{n\}, \sigma} \mathcal{E}_{\{n\}, \sigma} a_{\{n\}, \sigma}^{\dagger} a_{\{n\}, \sigma}$.

Taking the explicit expressions of spinors (21) into account and introducing the spinor functions

$$
\begin{aligned}
& \sum_{\sigma} \chi_{\sigma} \psi_{\sigma}(\mathbf{r})=\left(\begin{array}{c}
\cos \frac{\theta}{2} \psi_{+}-e^{-i \phi} \sin \frac{\theta}{2} \psi_{-} \\
e^{i \phi} \sin \frac{\theta}{2} \psi_{+}+\cos \frac{\theta}{2} \psi_{-}
\end{array}\right)= \\
& =\boldsymbol{\psi}(\mathbf{r})=\left(\begin{array}{c}
\psi_{\uparrow}(\mathbf{r}) \\
\psi_{\downarrow}(\mathbf{r})
\end{array}\right),
\end{aligned}
$$

the system of equations (28) or (35) can be written as a single stationary Pauli equation $\hat{H}_{\mathrm{P}} \boldsymbol{\psi}(\mathbf{r})=\mathcal{E} \boldsymbol{\psi}(\mathbf{r})$ with the Hamiltonian $\hat{H}_{\mathrm{P}}=H_{0}+\mathrm{V}_{\mathrm{SO}}$, where

$$
\begin{aligned}
& H_{0}=\frac{\hat{\mathbf{p}}^{2}}{2 m}+V(\mathbf{r}) \\
& \mathrm{V}_{\mathrm{SO}}=\frac{\lambda_{\mathrm{SO}}}{\hbar} \frac{\left(\hat{\mathbf{e}}+\mathbf{e}_{z}\right) \cdot[\nabla V(\mathbf{r}) \times \hat{\mathbf{p}}]}{1+\hat{\mathbf{e}} \mathbf{e}_{z}} \hat{\mathbf{e}} \hat{\boldsymbol{\sigma}} .
\end{aligned}
$$

In this case, the vector $\hat{\mathbf{e}}=\hat{\mathbf{u}}^{(0)} / u^{(0)}$, which enters the SOI operator and, by definition, characterizes a spin invariant, has to be consistent with the commutation condition for the matrix $\hat{\mathbf{u}}^{(0)} \hat{\boldsymbol{\sigma}}$ and the Hamiltonian. From whence, there arises the natural requirement

$$
\left[\hat{\mathbf{u}}^{(0)}, H_{0}\right]=0 \text {. }
$$

In other words, the vector operator $\hat{\mathbf{u}}^{(0)}$, which defines the generalized SOI operator, has to be an invariant (or a function of invariants) of the spatial motion in the given potential $V(\mathbf{r})$. In this case, every specific potential is connected with its "own" invariant (in the general case, not a single one). Therefore, condition (38) governs both the explicit expression for $\hat{\mathbf{u}}^{(0)}$ and, as a result, the form of the generalized SOI operator that contains not only the Thomas-Frenkel correction in this potential.

For illustration, let us consider below a model potential in the form of a QW, in which the bound electron states are formed by electrons captured by the well and moving freely in the well plane $(2 \mathrm{D}$ electrons). 


\section{Free 2D Electrons}

Let us analyze the electron states in a QW potential that are described by Eq. (35). Let us select the $z$-axis along the potential change direction, $V(\mathbf{r})=V(z)$, and represent the momentum operator in the form $\hat{\mathbf{p}}=\hat{\mathbf{p}}_{\perp}+\hat{p}_{z} \mathbf{e}_{z}$, where $\hat{\mathbf{p}}_{\perp}=\hat{p}_{x} \mathbf{e}_{x}+\hat{p}_{y} \mathbf{e}_{y}$. In this case, we have $\nabla V(z)=\mathbf{e}_{z} d V(z) / d z \equiv V^{\prime}(z) \mathbf{e}_{z}$, and Eq. (35) reads

$$
\begin{aligned}
& \left(\frac{\hat{\mathbf{p}}^{2}}{2 m}+V(z)+\sigma \frac{\lambda_{\mathrm{SO}}}{\hbar} V^{\prime}(z) \frac{\hat{\mathbf{e}} \cdot \mathbf{e}_{z} \times \hat{\mathbf{p}}}{1+\hat{\mathbf{e}} \mathbf{e}_{z}}\right) \times \\
& \times \psi_{\sigma}(\mathbf{r})=\mathcal{E} \psi_{\sigma}(\mathbf{r}) .
\end{aligned}
$$

It is easy to verify that two momentum components, $\hat{p}_{x}$ and $\hat{p}_{y}$, remain the integrals of motion for the chosen potential. Accordingly, the states of electrons captured by the QW will be characterized by a definite value of the momentum $\hat{\mathbf{p}}_{\perp}$ and will be described by the normalized wave function

$$
\begin{aligned}
& \psi_{\mathbf{k}_{\perp}}(\mathbf{r})=L^{-1} e^{i \mathbf{k}_{\perp} \mathbf{r}_{\perp}} \varphi_{\mathbf{k}_{\perp}}(z), \\
& \mathbf{r}_{\perp}=x \mathbf{e}_{x}+y \mathbf{e}_{y}, \\
& \mathbf{k}_{\perp}=k_{x} \mathbf{e}_{x}+k_{y} \mathbf{e}_{y},
\end{aligned}
$$

where $\mathbf{r}_{\perp}=x \mathbf{e}_{x}+y \mathbf{e}_{y}$ and $\mathbf{k}_{\perp}=k_{x} \mathbf{e}_{x}+k_{y} \mathbf{e}_{y}$. According to condition (38), the vector ê depends only on $\hat{\mathbf{p}}_{\perp}$. Substituting expression (39) into Eq. (35), we obtain the stationary one-dimensional SE

$$
\begin{aligned}
& \left(-\frac{\hbar^{2}}{2 m} \frac{d^{2}}{d z^{2}}+\frac{\hbar^{2} \mathbf{k}_{\perp}^{2}}{2 m}+V(z)+\right. \\
& \left.+\sigma \lambda_{\mathrm{SO}} \frac{\mathbf{e}\left(\mathbf{k}_{\perp}\right) \cdot \mathbf{e}_{z} \times \mathbf{k}_{\perp}}{1+\mathbf{e}\left(\mathbf{k}_{\perp}\right) \mathbf{e}_{z}} V^{\prime}(z)\right) \times \\
& \times \varphi_{\mathbf{k}_{\perp}, \sigma}(z)=\mathcal{E} \varphi_{\mathbf{k}_{\perp}, \sigma}(z),
\end{aligned}
$$

in which the fourth term in the parentheses on the left-hand side describes the generalized SOI. This equation coincides with the equation obtained in works $[6,11]$, in which an analytic general solution of the DE for the given problem was sought, as well as with the equation obtained in work [10] on the basis of the non-relativistic Hamiltonian (24). No additional conditions are imposed on the vector $\mathbf{e}\left(\mathbf{k}_{\perp}\right)$, which defines the electron spin state, so that it can be chosen with an arbitrary dependence on $\mathbf{k}_{\perp}$. This circumstance means that the free $2 \mathrm{D}$ electrons still retain a certain spin freedom, i.e. their states remain spin-indefinite.
The last term in the parentheses on the left-hand side of Eq. (40) characterizes the influence that the QW edges exert, by means of the SOI mechanism, on the dynamics of electrons, depending on their spin state. The vector $\mathbf{k}_{\perp}$ can be written in the form $\mathbf{k}_{\perp}=$ $=k_{\perp} \mathbf{e}_{k_{\perp}}$, where

$\mathbf{e}_{\mathbf{k}_{\perp}}=\frac{\mathbf{k}_{\perp}}{k_{\perp}}=\mathbf{e}_{x} \cos \varphi_{\mathbf{k}_{\perp}}+\mathbf{e}_{y} \sin \varphi_{\mathbf{k}_{\perp}}$.

In addition, let us consider expression (22), in which $\tan \varphi_{\mathbf{k}_{\perp}}=\frac{k_{y}}{k_{x}}, \quad k_{\perp}=\sqrt{k_{x}^{2}+k_{y}^{2}}$.

This procedure makes it possible to obtain the following expression for the coefficient that determines the magnitude of the generalized SOI in Eq. (40):

$\frac{\mathbf{e}\left(\mathbf{k}_{\perp}\right) \cdot \mathbf{e}_{z} \times \mathbf{k}_{\perp}}{1+\mathbf{e}\left(\mathbf{k}_{\perp}\right) \cdot \mathbf{e}_{z}}=k_{\perp} f\left(\mathbf{k}_{\perp}\right)$

where the function

$f\left(\mathbf{k}_{\perp}\right) \equiv f\left(\theta, \phi, \varphi_{\mathbf{k}_{\perp}}\right)=\frac{\sin \theta \sin \left(\phi-\varphi_{\mathbf{k}_{\perp}}\right)}{1+\cos \theta}$

was introduced. Generally speaking, the angles $\theta$ and $\phi$ may depend on the direction of the vector $\mathbf{k}_{\perp}$, i.e. $\theta=\theta\left(\mathbf{k}_{\perp}\right)$ and $\phi=\phi\left(\mathbf{k}_{\perp}\right)$.

When solving Eq. (40), the attention should be paid to that Hamiltonian (24) is a non-relativistic approximation, in which the terms of the order of $\lambda_{\text {SO }}$ were retained. Therefore, the solutions themselves will be correct exactly to this accuracy, which allows the term proportional to $\lambda_{\mathrm{SO}}$ to be considered as a perturbation. The solutions of the equation

$-\frac{\hbar^{2}}{2 m} \frac{d^{2} \psi(z)}{d z^{2}}+V(z) \psi(z)=\mathcal{E} \psi(z)$

are used as the zeroth approximation.

It is known that if $\psi_{n}(z)$ and $\mathcal{E}_{n}$ are eigenfunctions and eigenvalues, respectively, of Eq. (42), then the solutions of Eq. (40) obtained for the discrete QW spectrum in the first order of perturbation theory can be easily written in the form

$\varphi_{\mathbf{k}_{\perp}, \sigma}(z)=\psi_{n}(z)+\sigma \lambda_{\mathrm{SO}} k_{\perp} f\left(\mathbf{k}_{\perp}\right) \sum_{n^{\prime} \neq n} c_{n, n^{\prime}} \psi_{n^{\prime}}(z)$

$\mathcal{E}_{n, \sigma}\left(\mathbf{k}_{\perp}\right)=\mathcal{E}_{n}+\frac{\hbar^{2} \mathbf{k}_{\perp}^{2}}{2 m}+\sigma \lambda_{\mathrm{SO}} k_{\perp} v_{n n} f\left(\mathbf{k}_{\perp}\right)$.

ISSN 2071-0194. Ukr. J. Phys. 2019. Vol. 64, No. 6 
Here,

$c_{n, n^{\prime}}=\frac{v_{n n^{\prime}}^{*}}{\mathcal{E}_{n}-\mathcal{E}_{n^{\prime}}}$

$v_{n, n^{\prime}}=\int \psi_{n}^{*}(z) \frac{d V(z)}{d z} \psi_{n^{\prime}}(z) d z, \quad v_{n, n^{\prime}}^{*}=v_{n^{\prime}, n}$.

Thus, the 2D electrons are described by Hamiltonian (36), which takes the form

$\mathrm{H}_{2 D}=\sum_{n, \mathbf{k}_{\perp}, \sigma} \mathcal{E}_{n, \sigma}\left(\mathbf{k}_{\perp}\right) a_{n, \mathbf{k}_{\perp}, \sigma}^{\dagger} a_{n, \mathbf{k}_{\perp}, \sigma}$.

In this expression, the operators $a_{n, \mathbf{k}_{\perp}, \sigma}^{\dagger}\left(a_{n, \mathbf{k}_{\perp}, \sigma}\right)$ are the creation (annihilation) operators of "free" $2 \mathrm{D}$ electrons ${ }^{3}$ with the wave vector $\mathbf{k}_{\perp}$ in the $2 \mathrm{D} \mathcal{E}_{n, \sigma}\left(\mathbf{k}_{\perp}\right)$ band associated with the $n$-th discrete level in the QW, and the spin state of those electrons is determined by the vector $\mathbf{e}\left(\mathbf{k}_{\perp}\right)$ [see Eq. (22)].

From expressions (44) and (43), one can see that the generalized SOI gives rise, firstly, to the spin splitting (the Rashba effect) of 2D bands and, secondly, to the spin dependence of the electron density distribution over the QW thickness:

$\left|\varphi_{\mathbf{k}_{\perp}, \sigma}(z)\right|^{2}=\left|\psi_{n}(z)\right|^{2}+\sigma 2 \lambda_{\mathrm{SO}} k_{\perp} f\left(\mathbf{k}_{\perp}\right) F_{n}(z)$,

where

$F_{n}(z)=\sum_{n^{\prime} \neq n} \frac{\psi_{n}(z) w_{n, n^{\prime}} \psi_{n^{\prime}}(z)}{\mathcal{E}_{n}-\mathcal{E}_{n^{\prime}}}$.

The latter phenomenon has not been mentioned earlier. The spin splitting of $2 \mathrm{D}$ bands (44) by a magnitude of $2 \lambda_{\mathrm{SO}} k_{\perp} v_{n n} f\left(\mathbf{k}_{\perp}\right)$ is non-zero, only if the inverse potential symmetry is violated. The multiplier $v_{n n} \neq 0$ in front of $V_{0}(-z) \neq V_{0}(z)$ characterizes the QW asymmetry (both a probable inherent one and, e.g., that induced by an external electric field applied perpendicularly to the $x y$-plane). The spatial separation of densities for electrons with different spins is characterized by the function $2 \lambda_{\mathrm{SO}} k_{\perp} f\left(\mathbf{k}_{\perp}\right) F_{n}(z)$, which is finite in the symmetric QW as well, when the Rashba splitting is absent. Of course, this is a commonly known fact, and we report the corresponding results only as an evidence that the generalized SOI reproduces the well-known and recognized effects. In addition, it allows one to verify, to which of the spininvariants the potential should correspond in order that, e.g., the Rashba effect takes place.

\footnotetext{
3 In this case, the word "free" means that those particles are captured by the QW potential, but move freely in its plane.
}

\section{Manifestation of the Spin-Orbit Interaction in a 2D System}

It is well known that the bound electron states in a QW are described by a discrete non-degenerate spectrum of SE (42) with real-valued eigenfunctions $\psi_{n}(z)$ that are characterized by a definite parity. The even and odd functions alternate at that with the growth of their eigenenergies. If the QW is symmetric with respect to the coordinate origin, the matrix elements (45) differ from zero only between the even and odd states. Therefore, $F_{n}(-z)=-F_{n}(z)$, and the distribution of the probability to find an electron with the wave vector $\mathbf{k}_{\perp}$ and the spin number $\sigma$ turns out asymmetric as the electron shifts toward either of the QW edges. In this case, it is easy to see that the electrons with the same $\mathbf{k}_{\perp}$ but opposite spins become shifted to different surfaces, which directly testifies to the appearance of the spin Hall effect in this situation and, in essence, explains its mechanism.

At the same time, the spin splitting of energy bands and the spin-dependent electron distribution over the QW thickness depend on the QW form, which governs the explicit form of the solutions of Eq. (40) or (42). In this situation, the function $f\left(\mathbf{k}_{\perp}\right)$ plays its role and introduces an explicit dependence on the spin states of electrons into such phenomena. Really, according to Eq. (41), the arguments of this function are the spin (the angles $\theta$ and $\phi$ ) and spatial (the angle $\varphi_{\mathbf{k}_{\perp}}$ ) variables. The specific values of the spin variables $\theta$ and $\phi$ correspond to definite spin invariants, which are preserved in the field $V(z)$. In particular, if the $z$-component of the electric spin polarization $\epsilon_{z}$ is selected as the spin invariant, then $\theta=\pi / 2$ and $\phi=\pi / 2+\varphi_{\mathbf{k}_{\perp}}$ (state I). On the other hand, if the component of the spin pseudovector $\mathcal{S}_{j}$ in the $x y$ plane is selected as the spin invariant, then $\theta=\pi / 2$, whereas $\phi=$ const within the interval $0 \leq \phi \leq \pi / 2$ (state II; the value $\phi=0$ corresponds to the $x$ component of the invariant, and the value $\phi=\pi / 2$ to the $y$-component).

From definition (41), one can see that the influence of the obtained SOI on the dynamics of $2 \mathrm{D}$ electrons takes place, if the projection of $\mathbf{e}\left(\mathbf{k}_{\perp}\right)$ onto the direction of the unit vector $\mathbf{e}_{z} \times \mathbf{e}_{\mathbf{k}_{\perp}}$ differs from zero. This influence reaches its maximum, when $\mathbf{e}\left(\mathbf{k}_{\perp}\right)$ coincides with the latter vector (the Rashba spin state). The realization of a particular spin state has to be determined by the given conditions (the concentration of charge carriers, the presence of electric and/or mag- 
netic fields, the external pressure, the properties of specific interface, and so forth) and, therefore, has to manifest itself in real physical experiments.

As was noted in work [6], in an isolated 2D band (for example, when free electrons fill the ground state, $n=0$, QW level; only this case will be considered below), the total energy of $N_{e}$ electrons described by the equilibrium distribution function

$\bar{n}_{\sigma, \mathbf{k}}=\frac{1}{\exp \left\{\left(\mathcal{E}_{\sigma, \mathbf{k}}-\mu\right) / k_{B} T\right\}+1}=\bar{n}\left(\mathcal{E}_{\sigma, \mathbf{k}}\right)$

equals

$E_{\mathrm{tot}}=\sum_{\sigma, \mathbf{k}_{\perp}} \mathcal{E}_{\sigma}\left(\mathbf{k}_{\perp}\right) \bar{n}_{\sigma \mathbf{k}_{\perp}}, \quad \sum_{\sigma, \mathbf{k}_{\perp}} \bar{n}_{\sigma \mathbf{k}_{\perp}}=N_{e}$

and Rashba state I has the lowest energy in the absence of external fields and at the low temperature $T$. The Rashba splitting of $2 \mathrm{D}$ bands and their spin polarization were experimentally confirmed for a number of materials and structures, in which the charge carriers possess $2 \mathrm{D}$ properties (see, e.g., review [13]).

The spatial spin-separation of charge carriers in the QW can affect the observed local spin value $\mathbf{S}$ or the related magnetic moment $\mathbf{M}=(e / m c) \mathbf{S}$ that characterizes the $2 \mathrm{D}$ electron system. The spin per se, as was mentioned above, has no definite value for stationary states, because only its absolute value is the integral of motion. Therefore, the observed spin value is given by the average value of the spin operator. In the quantum field theory, the latter together with Hamiltonian (1) is given by the expression

$\mathbf{S}=\frac{\hbar}{2} \int \Psi^{\dagger}(\mathbf{r}) \hat{\boldsymbol{\Sigma}} \Psi(\mathbf{r}) d \mathbf{r}, \quad \hat{\boldsymbol{\Sigma}}=\left(\begin{array}{cc}\hat{\boldsymbol{\sigma}} & 0 \\ 0 & \hat{\boldsymbol{\sigma}}\end{array}\right)$

Here, the expressions for the bispinors are given in Eq. (9). Unlike the Hamiltonian, the operator $\mathbf{S}$ is not diagonalized in the free-particle representation, but contains terms that couple the creation and annihilation operators of electrons and positrons with different spin numbers. But, when calculating the average value $\langle\mathbf{S}\rangle=\operatorname{Sp}\left(\hat{\rho}_{e} \mathbf{S}\right)$ with the use of the statistical operator $\hat{\rho}_{e}$ for a system of electrons described by Hamiltonian (46), the non-diagonal terms vanish, and the following expression is obtained in the non-relativistic approximation for the spin density $\langle\hat{\mathbf{s}}\rangle=\langle\hat{\mathbf{S}}\rangle / L^{2}$ in the $\mathrm{QW}^{4}$ :

$\langle\hat{\mathbf{s}}\rangle=\int\langle\hat{\mathbf{s}}(z)\rangle d z$

where

$\langle\hat{\mathbf{s}}(z)\rangle=\frac{\hbar}{2 L^{2}} \sum_{\mathbf{k}_{\perp}, \sigma} \chi_{\sigma}^{\dagger} \hat{\boldsymbol{\sigma}} \chi_{\sigma}\left|\varphi_{\mathbf{k}_{\perp}, \sigma}(z)\right|^{2} \bar{n}_{\sigma, \mathbf{k}_{\perp}}$.

Here, $\bar{n}_{\sigma}, \mathbf{k}_{\perp}=\operatorname{Sp}\left(\hat{\rho}_{e} a_{\sigma}, \mathbf{k}_{\perp}^{\dagger} a_{\sigma}, \mathbf{k}_{\perp}\right)$ is the electron distribution function, and the spinors $\chi_{\sigma}$ are defined by expressions (21).

Now, by applying the explicit form of spinors, we obtain

$\chi_{\sigma}^{\dagger} \hat{\boldsymbol{\sigma}} \chi_{\sigma}=\sigma\left(\sin \theta \cos \phi \mathbf{e}_{x}+\sin \theta \sin \phi \mathbf{e}_{y}+\right.$

$\left.+\cos \theta \mathbf{e}_{z}\right)=\sigma \mathbf{e}\left(\mathbf{k}_{\perp}\right)$.

Passing from the summation over the quasicontinuous $\mathbf{k}_{\perp}$ variable to the integration, we arrive at the following expression for the average local magnitude of the spin vector density of electrons in the isolated 2D band:

$\langle\hat{\mathbf{s}}(z)\rangle=\frac{\hbar}{8 \pi^{2}} \int d k_{x} d k_{y} \sum_{\sigma} \sigma \mathbf{e}\left(\mathbf{k}_{\perp}\right)\left|\varphi_{\sigma, \mathbf{k}_{\perp}}(z)\right|^{2} \bar{n}_{\sigma, \mathbf{k}_{\perp}}$.

In a symmetric QW $\left(v_{n, n}=0\right)$, the spin splitting of the bands is absent, and the distribution function $\bar{n}_{\mathbf{k}_{\perp}}$ does not depend on the spin number. Taking this fact into account, after summing over the spin index and substituting the explicit expression for $\left|\varphi_{\sigma, \mathbf{k}_{\perp}}(z)\right|^{2}$, we obtain

$\langle\hat{\mathbf{s}}(z)\rangle=$

$\frac{\hbar}{2 \pi^{2}} \lambda_{\mathrm{SO}} \int_{0}^{2 \pi} d \varphi_{\mathbf{k}_{\perp}} \int_{0}^{\infty} d k_{\perp} \mathbf{e}\left(\mathbf{k}_{\perp}\right) k_{\perp}^{2} f\left(\mathbf{k}_{\perp}\right) \bar{n}_{\mathbf{k}_{\perp}} F(z)$.

One can see that the average value $\langle\hat{\mathbf{s}}(z)\rangle$ depends on the given electron spin state, which determines the specific values of the angles $\theta$ and $\phi$ in expressions (22) and (41). In the equilibrium 2D electron

4 To be more precise, in the non-relativistic approximation, when the quantities proportional to $\lambda_{\mathrm{SO}}$ are preserved, the Pauli matrix in this expression has to be substituted by the $\operatorname{matrix} \hat{\tilde{\boldsymbol{\sigma}}}=\hat{\boldsymbol{\sigma}}+2 \lambda_{\mathrm{SO}}(\boldsymbol{\Lambda}(\mathbf{k}) \times \hat{\boldsymbol{\sigma}}+\mathbf{k} \times[\mathbf{k} \times \hat{\boldsymbol{\sigma}}])$. Then the relativistic corrections along the spin direction, which is determined by an essentially non-relativistic expression, are small and can be neglected. 
gas characterized by the distribution function (47), we have $\bar{n}_{\mathbf{k}_{\perp}}=\bar{n}\left(\mathcal{E}_{\mathbf{k}_{\perp}}\right)$, and the electron spins become compensated over the whole QW thickness, $\langle\hat{\mathbf{s}}(z)\rangle=0$, in both indicated states I and II.

The situation changes radically, if an external electric field induces a current in the system. The external field, whose strength vector lies in the QW plane, perturbs the electron subsystem and changes its distribution function. In the linear approximation with respect to the perturbation, this function can be written as the sum [12]

$\bar{n}_{\mathbf{k}}^{\text {pert }}=\bar{n}\left(\mathcal{E}_{\mathbf{k}}\right)+\Delta n_{\mathbf{k}}$,

in which the correction associated with the field,

$\Delta n_{\mathbf{k}}=\frac{\hbar \tau e}{m} \mathbf{E} \cdot \mathbf{k}\left(-\frac{\partial \bar{n}\left(\mathcal{E}_{\mathbf{k}}\right)}{\partial \mathcal{E}_{\mathbf{k}}}\right)$

contains the phenomenological relaxation time $\tau$.

By selecting the $x$-axis along the field direction, we arrive at the following expression for the local spin polarization of charge carriers induced by the joint action of SOI and the electric current in the QW:

$\langle\hat{\mathbf{s}}(z)\rangle=\frac{\hbar^{2} \tau e E}{2 \pi^{2} m} \lambda_{\mathrm{SO}} \int_{0}^{2 \pi} d \varphi_{\mathbf{k}_{\perp}} \times$
$\times \int_{0}^{\infty} d k_{\perp} \mathbf{e}\left(\mathbf{k}_{\perp}\right) k_{\perp}^{3} f\left(\mathbf{k}_{\perp}\right) \cos \varphi_{\mathbf{k}_{\perp}}\left(-\frac{\partial \bar{n}\left(\mathcal{E}_{\mathbf{k}}\right)}{\partial \mathcal{E}_{\mathbf{k}}}\right) F(z)$.

Both the unit vector $\mathbf{e}\left(\mathbf{k}_{\perp}\right)$ and the function $f\left(\mathbf{k}_{\perp}\right)$ depend only on the angle $\varphi_{\mathbf{k}_{\perp}}$. Therefore, the integration over $k_{\perp}$ in this expression can be carried out by transforming it into the integration over the energy and taking into account that, at low temperatures, the derivative $(-\partial \bar{n}(\mathcal{E}) / \partial \mathcal{E})$ behaves like the $\delta$-function, $\delta\left(\mathcal{E}-\mathcal{E}_{\mathrm{F}}\right)$, where $\mathcal{E}_{\mathrm{F}}$ is the Fermi energy. In such a way, we obtain the average value

$\langle\hat{\mathbf{s}}(z)\rangle=\frac{\tau e E n_{e}}{\pi} \lambda_{\mathrm{SO}} F(z) \mathcal{I}$,

where $n_{e}$ is the number of electrons per unit QW area, $F(z) \equiv F_{0}(z)$, and the vector

$\mathcal{I}=\int_{0}^{2 \pi} \mathbf{e}(\theta, \phi) f\left(\theta, \phi, \varphi_{\mathbf{k}_{\perp}}\right) \cos \varphi_{\mathbf{k}_{\perp}} d \varphi_{\mathbf{k}_{\perp}}$

is determined by the spin-invariant, to which the electron states correspond, i.e. it depends actually on the

ISSN 2071-0194. Ukr. J. Phys. 2019. Vol. 64, No. 6 specific form of the general spin invariant $\hat{\mathcal{I}}_{\text {gen }}$, which was introduced above.

If the spin state is given by invariant $\mathrm{I}$, then the vector $\mathbf{e}\left(\mathbf{k}_{\perp}\right)=-\sin \varphi_{\mathbf{k}_{\perp}} \mathbf{e}_{x}+\cos \varphi_{\mathbf{k}_{\perp}} \mathbf{e}_{y}$ and $f\left(\mathbf{k}_{\perp}\right)=$ $=1$. From whence, we find that $\mathcal{I}=\pi \mathbf{e}_{y}$. For the spin state corresponding to invariant II, when $\mathbf{e}\left(\mathbf{k}_{\perp}\right)=$ $=\cos \phi \mathbf{e}_{x}+\sin \phi \mathbf{e}_{y}$ and $f\left(\mathbf{k}_{\perp}\right)=\sin \left(\phi-\varphi_{\mathbf{k}_{\perp}}\right)$, we obtain $\mathcal{I}=\pi\left(\cos \phi \mathbf{e}_{x}+\sin \phi \mathbf{e}_{y}\right) \sin \phi$. It is evident that the vector $\mathcal{I}=0$ at $\phi=0$, and the same vector $\mathcal{I}=\pi \mathbf{e}_{y}$ at $\phi=\pi / 2$.

Note that the external electric field directed along the $x$-axis lowers the system symmetry, and only the $y$-component of the spin pseudovector remains to be an invariant, $\phi=\pi / 2$. Thus, the local spin polarization of charge carriers in the QW is described by the formula

$\langle\hat{\mathbf{s}}(z)\rangle=\tau e E n_{e} \lambda_{\mathrm{SO}} F(z) \mathbf{e}_{y}$,

where $F(-z)=-F(z)$ and $F(0)=0$. Hence, an electric current in the QW layer induces the spin polarization of charge carriers near the boundary surfaces of the layer, with this polarization being opposite at the different surfaces. This, as was indicated above, is completely associated with the action of the generalized SOI. This phenomenon was called the spin Hall effect, and it was experimentally observed in structures with a similar geometry [14] (see also review [15]). In our case, it is a result of exclusively geometric properties of the examined $2 \mathrm{D}$ structures.

\section{Conclusions}

To summarize, in this work on the basis of the quantum theory of the Dirac spinor field, the generalized SOI operator $\mathrm{V}_{\mathrm{SO}} \sim \boldsymbol{\Lambda}(\hat{\mathbf{p}}, \boldsymbol{\nabla} V) \hat{\boldsymbol{\sigma}}$ is constructed. It is described by expressions (37) and (34). Its application to the non-relativistic SE provides a consistent description of the influence of SOI on electrons that move in an external potential $V(\mathbf{r})$. The latter is assumed to correspond to definite conditions of smallness in comparison with the characteristic electron energy (this criterion is always satisfied at least in the problems of solid state physics). In this case, the vector $\boldsymbol{\Lambda}(\hat{\mathbf{p}}, \boldsymbol{\nabla} V)$, which determines, in essence, SOI in addition to the well-known Thomas-Frenkel correction, also contains an additional contribution [see Eq. (16)].

Recall that the Thomas-Frenkel correction appears in vector (16), if the explicit dependence of the 
lower ("small") spinor on the momentum is made allowance for. On the other hand, the additional vector term $\boldsymbol{\Lambda}_{\mathrm{BEL}}\left(\mathbf{k}, \mathbf{k}^{\prime}\right)$ emerges owing to an analogous dependence of the $\Lambda_{\mathrm{SO}}$-order corrections to the upper ("large") spinor in the electron amplitude (bispinor). As to our knowledge, this term was ignored, as a rule.

It is extremely important that the spin invariants play a substantial role in finding vector (16). Some of them - for example, these are helicity operator and operator of the vector of electric spin polarization, as well as the operator of total momentum $\hat{\mathbf{J}}=\mathbf{L}+\frac{1}{2} \hbar \hat{\mathbf{\Sigma}}$ and the operator $\hat{K}=(\hat{\mathbf{L}} \hat{\mathbf{\Sigma}}+\hbar) \hat{\beta}$ commutating with it, where $\hat{\mathbf{L}}$ is the torque - correspond to the vectors $\mathbf{u}_{\nu}$ [see Eq. (10)] without relativistic corrections.

Finally, let us formulate conditions, under which the new, obtained in this work, correction to the standard and widely used SOI does not appear. It occurs, if the potential symmetry preserves one of the just indicated operators, and the generalized SOI operator automatically takes the form of the ThomasFrenkel correction for the corresponding spin state. If the potential symmetry does not violate the preservation of another invariant, then this spin state (or states) will be lost, if the term $\boldsymbol{\Lambda}_{\mathrm{BEL}}\left(\mathbf{k}, \mathbf{k}^{\prime}\right)$ in Eq. (16) is ignored. This was unambiguously illustrated by the example of a $\mathrm{QW}$ potential, in which the Thomas-Frenkel correction can describe only the spin state with the Rashba splitting. However, it was shown that this correction is not the only possible one. Currently, we may only hope for that not only the Thomas-Frenkel term is responsible for all possible "spin" consequences of SOI, and that the generalized SOI will find its application.

The authors are grateful to Yu. B. Gaididei for discussing the results obtained, in particular, the mechanism of the spin Hall effect. The work was carried out in the framework of the budget program KPKVK 6541230 and the scientific program 0117 U00236 of the Department of Physics and Astronomy of the National Academy of Sciences of Ukraine.

1. H.A. Bethe. Intermediate Quantum Mechanics (W.A. Benjamin, 1964).

2. A.S. Davydov. Quantum Mechanics (Pergamon Press, 1976).

3. L.H. Thomas. The motion of the spinning electron. Nature 117, 514 (1926).

4. J. Frenkel. Zur Theorie der Elastizitätsgrenze und der festigkeit kristallinischer Körper. Z. Phys. 37, 243 (1926).
5. A. Eremko, L. Brizhik, V. Loktev. Spin states of Dirac equation and Rashba spin-orbit interaction. Ann. Phys. 361, 423 (2015).

6. A. Eremko, L. Brizhik, V. Loktev. General solution of the Dirac equation for quasi-two-dimensional electrons. Ann. Phys. 369, 85 (2016).

7. N.N. Bogoliubov, D.V. Shirkov. Quantized Fields (Nauka, Moscow, 1980) (in Russian).

8. V.B. Berestetskii, E.M. Lifshitz, L.P. Pitaevskii. Relativistic Quantum Theory (Pergamon Press, 1982).

9. A.A. Sokolov, I.M. Ternov. Relativistic Electron (Nauka, 1974) (in Russian).

10. A.A. Eremko, L.S. Brizhik, V.M. Loktev. On the theory of the full set of relativistic corrections for Schrödinger equation. Low Temp. Phys. 44, 734 (2018).

11. A.A. Eremko, V.M. Loktev. On the theory of eigen spin states and spin-orbit interaction of quasi-two-dimensional electrons. Fiz. Nizk. Temp. 43, 456 (2017) (in Russian).

12. J. M. Ziman. Principles of the Theory of Solids (Cambridge Univ. Press, 1979).

13. G. Bihlmayer, J. Rader, K. Winkler. Focus on the Rashba Effect. New J. Phys. 17, 050202 (2015).

14. Y.K. Kato, R.C. Myers, A.C. Gossard, D.D. Awschalom. Observation of the spin Hall effect in semiconductors. Science 306, 1910 (2004).

15. J. Sinova, S.O. Valenzuela, J. Wunderlich, C.H. Back, T. Jungwirth. Spin Hall effects. Rev. Mod. Phys. 87, 1213 (2015).

Received 01.02.19.

Translated from Ukrainian by O.I. Voitenko

О.О. Еремко, Л.С. Брижик, В.М. Локтев

УЗАГАЛЬНЕНА СПІН-ОРБІТАЛЬНА

ВЗАЄМОДІЯ ТА ІІЇ ПРОЯВ У ДВОВИМІРНИХ

ЕЛЕКТРОННИХ СТРУКТУРАХ

$\mathrm{P}$ е $з$ ю м е

В рамках квантової теорії поля Дірака, що описує електрони і позитрони як елементарні збудження спінорного поля, переходом до нерелятивістського наближення в операторі Гамільтона спінорного поля з урахуванням наявності зовнішнього потенціалу знайдено узагальнений оператор спінорбітальної взаємодіі. Показано, що цей оператор містить окрім відомих доданків також новий внесок. На прикладі модельного потенціалу у вигляді квантової ями показано, що рівняння Шредінгера з таким узагальненим оператором спін-орбітальної взаємодії описує всі спінові стани, одержані з самого рівняння Дірака. Досліджено залежність спінорбітальної взаємодії від спінового стану у квазідвовимірних локалізованих в площині квантової ями електронних системах. Показано, що електричний струм у шарі квантової ями індукує спінову поляризацію носіїв поблизу граничних поверхонь шару з протилежною поляризацією на протилежних поверхнях, що цілком зумовлено дією узагальненої спін-орбітальної взаємодії і відомо як спіновий ефект Холла, що і спостерігалося експериментально у структурах з подібною геометрією. 